Research Paper

\title{
SiRNA-Mediated RRM2 Gene Silencing Combined with Cisplatin in the Treatment of Epithelial Ovarian Cancer In Vivo: An Experimental Study of Nude Mice
}

\author{
Ting Xue ${ }^{1}$, Liming Wang ${ }^{2}$, Yong $\mathrm{Li}^{2}$, Hao Song ${ }^{3}$, Huijun $\mathrm{Chu}^{2}$, Hongjuan Yang ${ }^{2}$, Ailian Guo ${ }^{1}$, Jinwen Jiao ${ }^{2}$ \\ 1. Qingdao University, Qingdao, China. \\ 2. Department of Obstetrics and Gynecology, The Affiliated Hospital of Qingdao University, Qingdao, China. \\ 3. Department of Oncology, The Affiliated Hospital of Qingdao University, Qingdao, China.
}

$\square$ Corresponding author: Jinwen Jiao, Department of Obstetrics and Gynecology, The Affiliated Hospital of Qingdao University, No. 1677, Wutai Mountain Road, Huangdao District, Qingdao 266000, China. Phone: 18661806023; Fax: +86 0532 82919608; E-mail: jiaojw@qduhospital.cn

(1) The author(s). This is an open access article distributed under the terms of the Creative Commons Attribution License (https://creativecommons.org/licenses/by/4.0/). See http://ivyspring.com/terms for full terms and conditions.

Received: 2019.02.11; Accepted: 2019.08.24; Published: 2019.10.21

\begin{abstract}
Introduction: We aimed to explore small interfering (si)RNA silencing of ribonucleotide reductase $\mathrm{M} 2$ (RRM2) gene combined with cisplatin for the treatment of human ovarian cancer in nude mice models of subcutaneous transplantation of tumor cells.

Methods: After conventional cultivation of human ovarian cancer cell line SKOV3 in vitro, SKOV3 cells were injected into the right back of nude mice by subcutaneous injection to establish the subcutaneous tumor models. Twenty-four tumor-burdened rats were randomly divided into four groups $(n=6)$ : siRNA group, siRNA in combination with cisplatin group, cisplatin group, and control group. Intraperitoneal injection of cisplatin and subcutaneous injection of siRNA were performed weekly. Tumor volume was measured, and tumor growth inhibition rate was calculated. RRM2 expression at the mRNA and protein levels was detected by reverse transcription-polymerase chain reaction and immunohistochemistry.

Results: In the siRNA group, the tumor volume and tumor growth inhibition rate were $249.60 \pm 20.46$ $\mathrm{mm}^{3}$ and $36.39 \%$, respectively. The tumor growth inhibition rate and tumor volume were significantly different between the siRNA and control groups $(p<0.05)$. In the cisplatin group, the tumor volume and tumor growth inhibition rate were $249.86 \pm 12.46 \mathrm{~mm}^{3}$ and $41.10 \%$, respectively. The tumor growth inhibition rate and tumor volume were significantly different between the cisplatin and control groups $(p<0.05)$. In the siRNA + cisplatin group, the tumor volume reduced to $180.84 \pm 16.25 \mathrm{~mm}^{3}$ and the tumor growth inhibition rate was increased to $64.33 \%$, which were significantly different compared with the control group $(\mathrm{p}<0.01)$. Significant downregulation of RRM2 mRNA and protein expression in the tumor tissues was detected by reverse transcription polymerase chain reaction and immunohistochemistry assay $(\mathrm{p}<0.05)$.

Discussion: siRNA alone or combined with cisplatin can effectively inhibit the growth of human ovarian cancer in nude mice models of subcutaneous transplantation of tumor cells. RRM2 gene silencing may be a potential treatment regimen for ovarian cancer in future.
\end{abstract}

Key words: RRM2, siRNA/RNAi, cisplatin, ovarian cancer, nude mice

\section{Introduction}

Ovarian cancer is one of the three most common malignancies of the female reproductive system, with the highest mortality rate among all gynecological tumors[1]. Surgery, combined with platinum- or paclitaxel-based chemotherapy, is the main treatment for patients with ovarian carcinoma. However, patients with ovarian carcinoma easily develop drug resistance[2], such as cisplatin resistance. These drugresistant patients generally have few treatment options. Therefore, there is an urgent need for the identification of novel therapeutic strategies targeting drug-resistant mechanisms to enhance cisplatin's 
killing effect on tumor cells and to increase sensitivity to chemotherapy.

Gene therapy refers to the introduction of exogenous genes into target cells to correct or compensate for diseases caused by genetic defects or abnormal gene expression. Innovations of gene therapy technology and clinical trials have increased in recent years, and a number of gene therapy projects have been approved and listed in the United States, China, and other countries to treat diabetes, cardiocerebrovascular disease, rheumatism, and various types of cancer[3-9]. Ribonucleotide reductase $(R R)$ is a potential therapeutic target for cancer because its role in catalytic reduction is necessary for DNA replication and repair[10]. It is the rate-limiting enzyme in the conversion of ribonucleotide 5'-diphosphates into 2'-deoxyribonucleotides. Human $R R$ consists of two parts: RRM1 and RRM2. Unlike $R R M 1, R R M 2$ is only expressed during the late G1/ early $S$ phase of the cell cycle, when DNA replication occurs[11]. Over expression of RRM2 plays a positive role in tumor growth. Elevated RR activity and over expression of $R R M 2$ significantly increase the drugresistant properties and the angiogenesis of human cancer cells[12]. RRM2 was identified as a diagnostic marker of several cancers, suggesting that $R R M 2$ is a potential therapeutic target. Therefore, an anti-tumor strategy that interferes with the activity of RRM2 has the potential to inhibit the growth of ovarian cancer. In our previous study[13], our results suggested that small interfering RNA(siRNA)-mediated RRM2 knockdown significantly reversed SKOV3/DDP cell resistance to cisplatin. Choosing an efficient gene delivery system has been a major challenge for gene therapy. We used Lipofectamine 2000 to effectively transfer siRNA into SKOV3/DDP cells. Previously, we have demonstrated the synergistic inhibitory effect of RNA interference technology combined with gemcitabine and cisplatin in SKOV3/DDP cells; however, no study has explored whether RRM2 gene therapy can also reverse ovarian cancer resistance to cisplatin in vivo. Here, we used the human ovarian carcinoma SKOV3 cell line to construct a nude mouse subcutaneous transplantation model to investigate whether RRM2 gene therapy was a novel therapeutic option for the treatment of epithelial ovarian cancer.

\section{Methods}

\section{Cell culture}

SKOV3 cell lines were purchased from the Cell Resource Center of the Shanghai Institute of Life Sciences and preserved by our laboratory. They were cultured in DMEM-F12 medium supplemented with 5\% FBS, $100 \mu \mathrm{g} / \mathrm{mL}$ streptomycin, $100 \mathrm{U} / \mathrm{mL}$ penicillin, and $2 \mathrm{mM}$ L-glutamine at $37^{\circ} \mathrm{C}$ in an incubator containing $5 \% \mathrm{CO}_{2}$.

\section{siRNA duplexes}

siRNA targeting RRM2 -(sense: 5'-GGAGC GAUUUAGCCAAGAATT-3'; antisense: 5'-UUCUUG GCUAAAUCGCUCCTT-3') was purchased from GenePharma (Shanghai, China) and a negative control siRNA was a gift from them.

\section{Lipofectamine transfection}

Cells were seeded in 24-cell plates 24 hours before transfection in medium containing $10 \%$ FBS, so that they reached about $50 \%$ confluency. siRNA was complexed with Lipofectamine 2000 (Invitrogen, Carlsbad, CA, USA) according to the manufacturer's instructions and was applied to each control plate. These cells were divided into four groups: the blank group, the liposome group, the non-targeting siRNA group and the targeting siRNA group. Transfection media was removed and replaced with new media after 4 hours. Cells were collected after 72 hours and RNA was extracted for analysis.

\section{Animal procedures and treatment}

All animal procedures were conducted in accordance with institutional and national guidelines. All experimental protocols were approved by the Animal Care and Welfare Committee of the Affiliated Hospital of Qingdao University. (License NO. AHQU20170914A) Female BALB/c nude mice (aged 4 weeks) were purchased from SHANGHAI SLAC and housed under specific pathogen-free conditions at the laboratory animal room for a week before the experiment. All of the mice were inoculated with a subcutaneous injection of $2 \times 10^{7}$ cells plus PBS in the right dorsum (injection volume $=200 \mu \mathrm{L}$ ). The sizes of tumors were measured from the first day until the day of death after cell injection using calipers with the formula: $\mathrm{V}$ (volume) $=1 / 2 \times a \times b^{2}$, where " $a$ " represents the greatest length and " $b$ " represents the perpendicular width[14]. Furthermore, tumor growth inhibition rate was calculated as: Tumor growth inhibition rate $(\%)=($ tumor volume in control group tumor volume in treatment group) / tumor volume in control group $\times 100 \%$. When palpable tumors had developed at the sites of injection $\left(>50 \mathrm{~mm}^{3}\right)$, tumor-bearing animals were randomly allocated to four groups $(n=6)$ and were treated with DNase/RNase-free water, cisplatin ( $3 \mathrm{mg} / \mathrm{kg})$, physiological saline, and siRNA-RRM2 (500 pmol) via intraperitoneal and subcutaneous injection after tumor inoculation, the specific administration methods of the four treatment groups were shown (Figure 1). Drug treatment was performed weekly for 4 weeks. All mice were sacrificed by cervical vertebra 
dislocation at 24 days after first dosage. Tumors were harvested and immobilized with $4 \%$ neutral paraformaldehyde and frozen with liquid nitrogen immediately. Tumor volume, number of nodules, and nude mouse weight were recorded every three days.

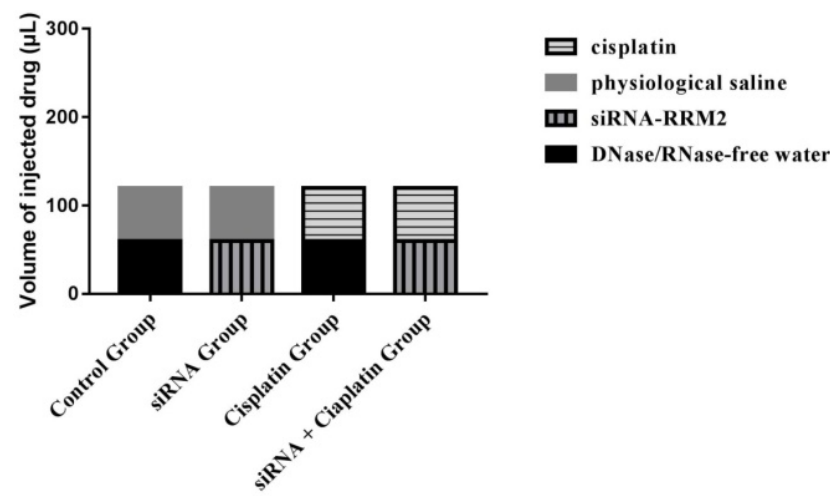

Figure 1. The specific administration methods of the four treatment groups.

\section{Reverse transcription-polymerase chain reaction}

Total RNA was extracted from the transfected cells and tumor tissues using RNAiso PLUS. The sample was reverse transcribed using a TaKaRa RNA PCR Kit (AMV) Version.3.0 (TaKaRa, Beijing, China). The GAPDH gene was used as an endogenous control. Primers were synthesized by Sangon Biotech (Shanghai, China) as follows: RRM2-Forward: 5'-GCGATTT AGCCAAGAAGTTCAGAT-3', RRM2-Reverse: 5'-CC CAGTCTGCCTTCTTCTTGA-3'; GAPDH-Forward:5'TCACTGCCACCCAGAAGACT-3', GAPDH-Reverse: 5'-TTCTAGACGGCAGGTCAGGT-3'. The reverse transcription-polymerase chain reaction process contained a step at $94^{\circ} \mathrm{C}$ for $180 \mathrm{~s}$, followed by $30 \mathrm{~s}$ at $94^{\circ} \mathrm{C}, 30 \mathrm{~s}$ at $57^{\circ} \mathrm{C}$, and $45 \mathrm{~s}$ at $72^{\circ} \mathrm{C}$ for 32 cycles, followed by analysis.

\section{Immunohistochemistry}

To detect intracellular localization and expression levels of $R R M 2$, we used rabbit antihuman RRM2 antibody (Abcam, ab209995, Tris-EDTA buffer) as the primary antibody, then combined it with a secondary antibody. Cell nuclei were counterstained using 4,6-diamidino-2-phenylindole (DAPI, Invitrogen). All tissue slides were evaluated and scored by a qualified pathologist. The expression of $R R M 2$ was determined by cytoplasmic staining intensity and positive cell rate. According to the staining intensity, the results were as follows: no staining (0), weak staining (1), medium staining (2), and strong staining (3). The positive cell rate was graded as $<5 \%$ (0), $6 \% \sim 25 \%$ (1), $26 \%-50 \%$ (2), and $>50 \%$ (3). The final score is the sum of the above two scores.

\section{Statistical analysis}

All data were presented as mean values \pm standard deviation. The statistical significance was evaluated by one-way analysis of variance when all groups were compared, and Tukey's HSD for post-hoc analysis between two groups. In all tests, differences were considered to be statistically significant at $\mathrm{p}<0.05$.

\section{Results}

\section{RNA interference experiments in vitro}

The expression levels of $R R M 2$ were examined with reverse transcription polymerase chain reaction in SKOV3 cells (Figure 2). RRM2 mRNA was higher from the liposome group, the non-targeting siRNA group and the blank group than in the targeting siRNA groups $(\mathrm{p}<0.05)$. There was no significant difference between the blank and non-targeting siRNA groups $(p>0.05)$.

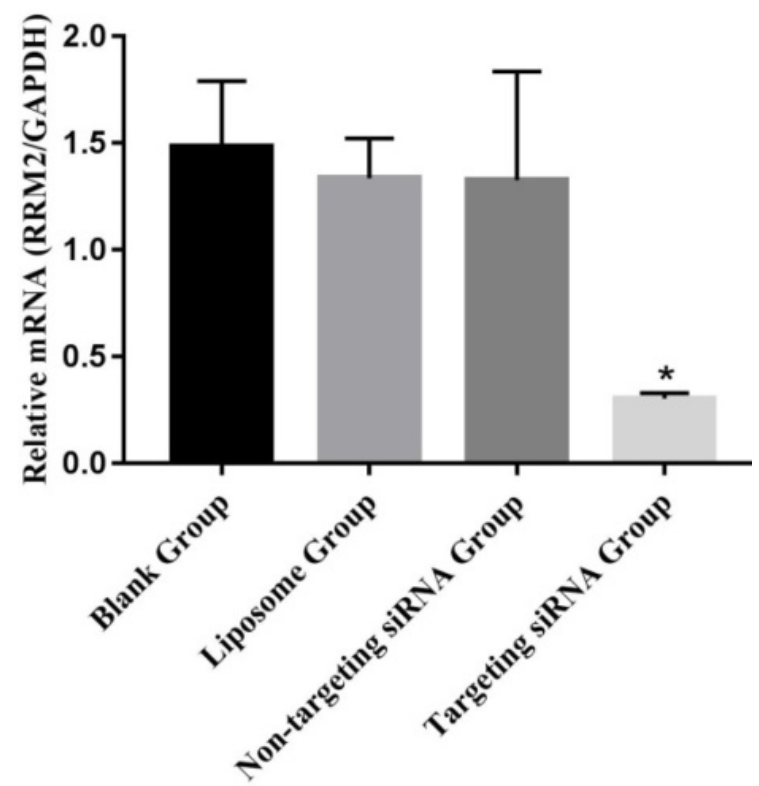

Figure 2. Expression of RRM2 mRNA in SKOV3 cells. Relative mRNA level of RRM2 in SKOV3 cells were analyzed by reverse transcription-polymerase chain reaction with GAPDH as a control, ${ }^{*} \mathrm{P}<0.05$ as compared with the blank group.

\section{Tumor volume and tumor growth inhibition of subcutaneous transplanted tumors}

Although treatment with siRNA or siRNA + cisplatin significantly suppressed tumor growth compared with that in the control group, the optimal therapeutic effect on tumor growth was achieved by siRNA + cisplatin treatment (Figure 3). The suppression of tumor growth in siRNA + cisplatin mice continued until the day of sacrifice, reaching a mean volume of $180.84 \mathrm{~mm}^{3}$, while tumors of mice treated with control, cisplatin, or siRNA grew persistently with mean tumor volumes of $342.13 \mathrm{~mm}^{3}$, 
$249.86 \mathrm{~mm}^{3}$, and $249.60 \mathrm{~mm}^{3}$, respectively $(\mathrm{p}<0.05)$. Furthermore, the combined treatment of siRNA and cisplatin caused marked tumor growth suppression compared with siRNA alone $(\mathrm{p}<0.05)$, but there was no significant difference between the siRNA and cisplatin groups ( $p>0.05$; Figure 4$)$.

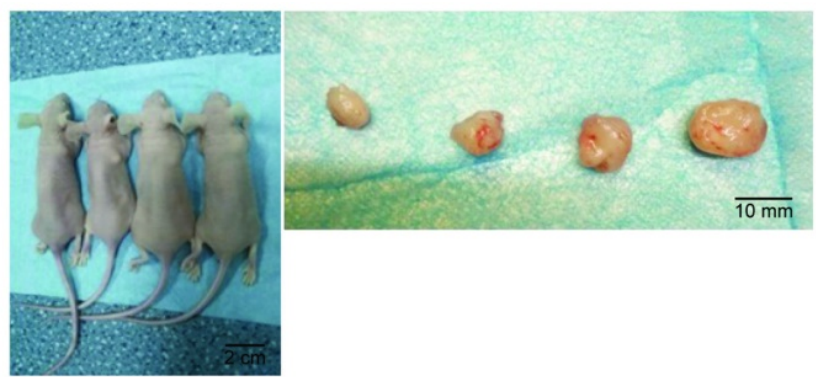

Figure 3. Model of subcutaneous transplanted tumors in nude mice. Subcutaneous transplanted tumors (left) in a whole animal and excised tumor tissues (right). From left to right: siRNA+ cisplatin group, cisplatin group, siRNA group, and control group.

\section{Pathological sections of tumor tissue}

After the nude mice were sacrificed, fresh tumors were excised. The tissue was fixed in formaldehyde solution and routinely made into paraffin sections. Histological examination with hematoxylin and eosin staining of tumor tissues showed necrotic cells along with tissue disorganization, with large tumor cells, large and hyperchromatic nuclei, prominent nucleoli, and obvious mitotic images in all treatment groups, especially in the siRNA + cisplatin group (Figure 5A). Immunohistochemistry showed expression of RRM2 in the transplanted tumor tissues of each group, and cell staining was observed in each treatment group. The positive cells exhibited yellowish brown granules in the cytoplasm. The siRNA, cisplatin, and siRNA + cisplatin groups showed incomplete cytoplasmic expression, which was significantly higher in the control group (Figure 5B).

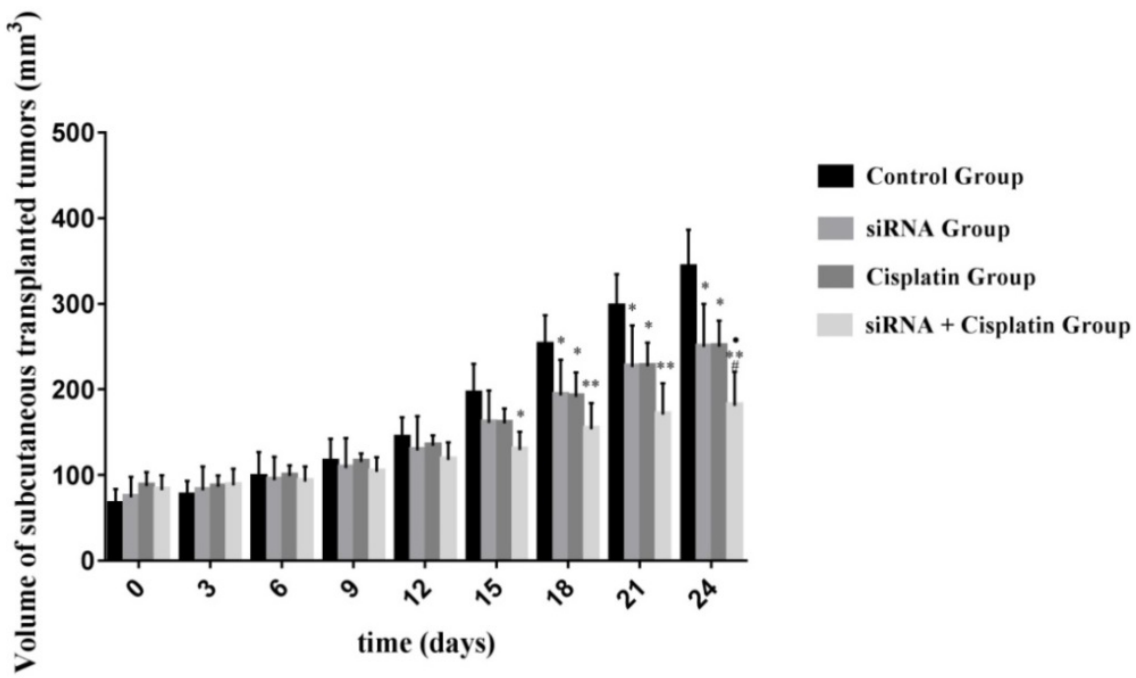

Figure 4. Volume of subcutaneous transplanted tumors on Nude Mouse at different time points. The sizes of tumors were measured from the first day until the day of death after cell injection using calipers with the formula. ${ }^{*} \mathrm{P}<0.05$, ${ }^{* *} \mathrm{P}<0.001$ as compared with control group. ${ }^{*}<0.05$ as compared with siRNA group. $\# \mathrm{P}<0.05$ as compared with cisplatin group.

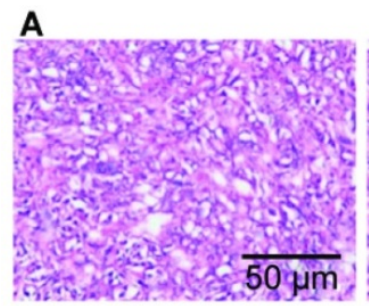

B

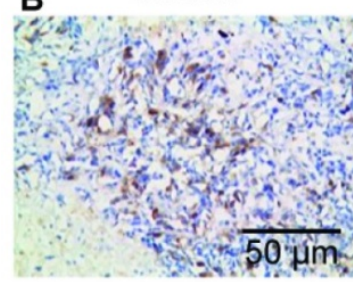

Control

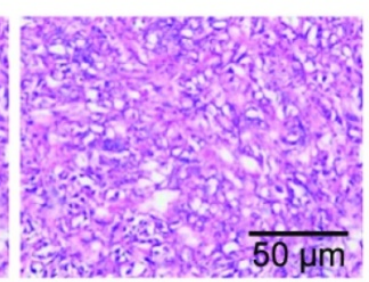

SiRNA

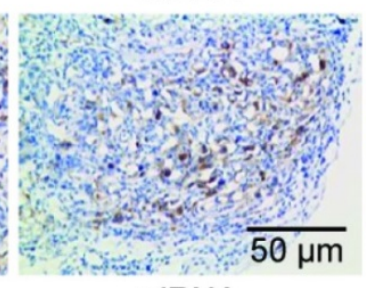

SiRNA

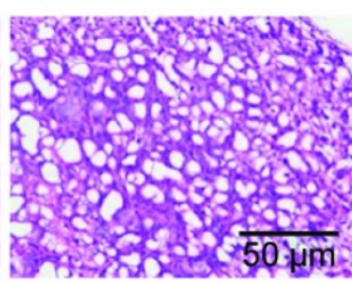

Cisplatin

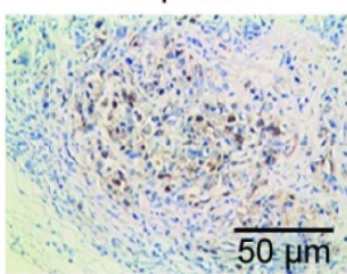

Cisplatin

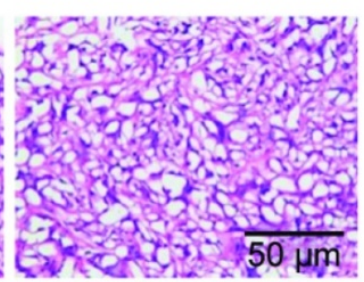

SiRNA + Cisplatin

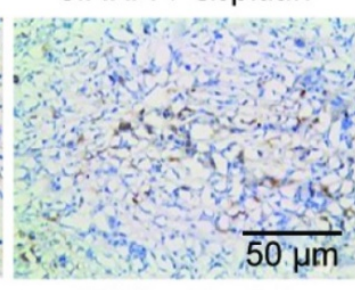

SiRNA + Cisplatin

Figure 5. Pathological section of tumor tissue Histological examination with hematoxylin and eosin staining (A) and immunohistochemical staining (B) of tumor tissue. 


\section{Effects of RRM2 with cisplatin therapy on expression of RRM2 $\mathrm{mRNA}$ and protein in subcutaneous transplanted tumors}

To determine the potential mechanism of cell growth inhibition in subcutaneous transplanted tumors, the expression of RRM2 mRNA and protein was examined. The gene and protein expression levels of RRM2 were examined with reverse transcription polymerase chain reaction (Figure 6A) and immunohistochemical staining (Figure 6B) in subcutaneous transplanted tumors after different treatments. RRM2 mRNA and protein expression was lower in tumors from the siRNA and siRNA + cisplatin groups than in the tumors of mice in the control groups and was significantly lower in the tumors of mice treated with siRNA + cisplatin, than in those from any other group $(\mathrm{p}<0.05)$. Compared with the control group, the mRNA and protein expression of RRM2 in cisplatin group were lower $(\mathrm{p}<0.05)$. There was no significant difference between the siRNA and cisplatin groups ( $p>0.05)$.

\section{Discussion}

Ovarian cancer is a malignant tumor that seriously endangers women's health. It has the highest mortality rate among gynecological tumors. Extensive pelvic and abdominal implantation and metastasis can occur in the early stages. Gene therapy is a new technology developed in recent years. As one of them, RNA interference mainly uses doublestranded RNA to specifically mediate the degradation of its complementary homologous mRNA series, so it can specifically inhibit the expression of the target protein with strong inhibition and high specificity. The combination of gene and chemotherapeutic drugs presents a promising therapeutic strategy for effective
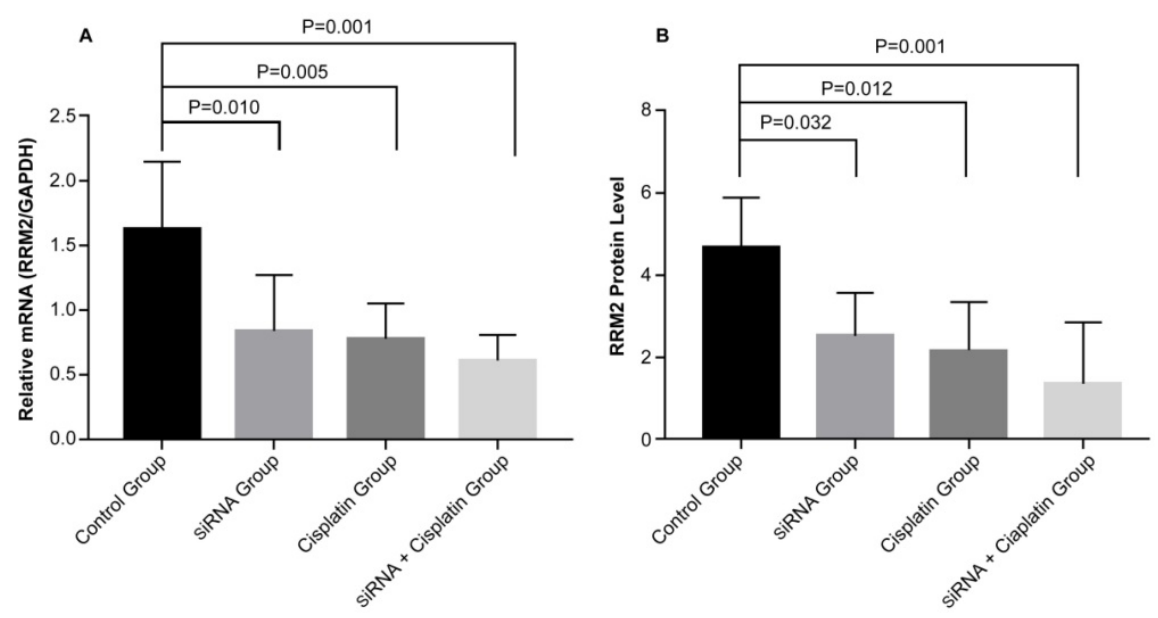

Figure 6. Expression of RRM2 mRNA and related proteins in subcutaneous transplanted tumors. Relative mRNA level of RRM2 in tumor tissues were analyzed by reverse transcription-polymerase chain reaction (A) with GAPDH as a control. The protein expression of RRM2 in tumor tissue was assessed by immunohistochemical staining (B). All data were representative of three independent experiments. cancer treatment[15-18]. RRM2 is not only a potential molecular marker of many malignant tumors, but also can disrupt the growth and differentiation of normal cells, thus playing the role of oncogenes[7, 19, 20]. $R R M 2$ interacts with many oncogenes to determine the potential for cell transformation and tumorigenesis. Intracellular RRM2 expression and enzyme activity are positively correlated with tumor resistance, invasion, and migration[21, 22]. Overexpression of RRM2 can promote the proliferation, invasion, and drug resistance of oncogenes and increase the metastasis of tumor cells. In contrast, downregulation or silencing of the expression of RRM2 can lead to apoptosis of malignant tumor cells, thereby inhibiting cell proliferation, metastasis, and reversing cell resistance[8]. Therefore, the expression level and activity of RRM2 are closely related to the proliferation of tumor cells and may play a decisive role in the mechanism of controlling the invasion and development of malignant tumors.

In this study, we provided a novel strategy for ovarian carcinoma. Here, we used ovarian cancer cell line SKOV3 cells to construct subcutaneous transplanted tumor model. siRNA is used to treat tumor as a monotherapy or in combination with cisplatin. The relative low dose of cisplatin used in the present study did not produce obvious toxic effects, while those of siRNA are not yet clear. However, these levels were able to inhibit tumor growth in subcutaneous transplanted tumor when combined with RRM2 gene therapy. High expression of RRM2 is common in cancer and is also associated with resistance to chemotherapy and radiotherapy[23, 24]. Thus, this phenomenon led us to hypothesize that the expression level of RRM2 is involved in the acquisition and development of resistance to multiple drugs. In a previous study, the increased sensitivity of SKOV3/DDP cells to cisplatin drugs after transfection with RRM2 siRNA further demonstrated that RRM2 may be an important mediator of cisplatin- mediated resistance[13]. We also further investigated the role of RRM2 in tumor growth inhibition in subcutaneous transplanted tumors. In our study, compared with other groups, the expression of RRM2 mRNA and protein and subcutaneous transplanted tumors volume were the lowest after siRNA + cisplatin treatment of mouse tumors. 
Resistance to apoptosis is a major reason for the failure of treatment of malignancies. Indeed, decreased RRM2 levels induced by RRM2 gene therapy may activate apoptosis pathways and inhibit cisplatin-induced DNA damage repair[23], thereby promoting the apoptosis process initiated by anti-cancer agents. RRM2 plays a key role in the regulation of DNA synthesis and cell proliferation in the DNA replication stage, mainly in the late G1 or early $S$ phase of the cell cycle[25, 26]. In our study, we speculated that the slow growth of tumor volume in the siRNA + cisplatin group was related to the role of $R R M 2$. On the one hand, the expression of RRM2 was reduced, which arrest cell cycle at G1/S period and eventually induced necrosis. On the other hand, cisplatin leads to DNA damage. Inhibition of RRM2 may reduce the DNA repair ability through blocking DNA repairing or lead to apoptosis when DNA repair is failed. The resistance to cisplatin is associated with the cell's increased ability of DNA repair. The combined treatment of siRNA and cisplatin caused marked tumor growth suppression compared with that of cisplatin alone, which may be related to siRNA increasing the sensitivity of tumor cells to cisplatin chemotherapy. One of the major problems of malignant progression is induction of invasion and migration. In addition to participating in DNA synthesis, RRM2 also has an impact on the potential biological behavior and metastasis of malignant tumors and the generation of tumor drug resistance. If $R R M 2$ gene therapy combined with chemotherapy is an effective method for increasing the sensitivity of resistant cancer cells to chemotherapeutic agents, it will efficiently reduce the recurrence of invasion and migration. Previous studies have demonstrated that the RRM2 gene plays a role in regulating tumor cell proliferation in different cancers and that decreased expression of RRM2 increases sensitivity to temozolomide, gemcitabine, and platinum-based antitumor drugs[11, 25, 27]. Furthermore, studies have shown that several signal transduction pathways (such as VEGF, MMP2, and MMP9) are also associated with invasion and migration. Most human tumors overexpress VEGF, which is known to be a highly regulated angiogenic factor in cancer development[28-30]. Overexpression of RRM2 increased VEGF expression. Knockdown of RRM2 by siRNA may potentially inhibit cancer angiogenesis. MMPs involved in the cleavage of cell surface receptors possess gelatinase activity to enhance cancer invasion and metastasis[31-33].

In this study, there was no significant injury to the lungs, kidney, or other important organs after the intraplastic injection of siRNA-RRM2 directly into a transplanted tumor, indicating that siRNA-RRM2 does not cause serious adverse reactions. However, the invasion of tumor cells was not tested in this study. Cunjian et al[34]. Subcutaneously inoculated an ovarian cancer cell line, SKOV3/DDP, into the necks of nude mice, and, in certain mice, metastatic lesions were found in the abdominal organs, including the liver and mesentery. Invasion and metastasis of tumor cells are mainly determined by their biological characteristics, as well as by factors such as the local microenvironment and host immunity. Tumor-bearing mice are mutant mice with congenital thymus defects. $\mathrm{T}$ lymphocyte development is blocked, resulting in $\mathrm{T}$ lymphocyte immune deficiency. The spleen is a key immune organ of nude mice, and other organs, such as the liver, also have a large number of macrophages, but no obvious tumor cell infiltration was found in our study.

As a limitation of our study, demonstrating the efficacy of this treatment in one cell line does not fully demonstrate its efficacy in other ovarian cancer cell lines expressing RRM2. Although our previous studies have shown that RRM2 gene therapy may reduce the proliferation of SKOV3 cells in vivo and lead to an increase in apoptosis, the role of RRM2 gene therapy in different ovarian cancer types still requires further research. Furthermore, methods for ensuring high efficiency, stability, and safety of gene therapy; selecting the appropriate transporter; and applying this research to clinical practice remain to be determined.

In conclusion, our study suggests that in a mouse subcutaneous transplanted tumor model, expression of RRM2 mRNA and protein and the volume of subcutaneous transplanted tumors were the lowest in tumors of mice treated with siRNA + cisplatin. These results enhance our current understanding of the role of $R R M 2$ in tumor growth and provide new avenues for the development of effective treatment and prevention of ovarian cancer.

\section{Abbreviations}

RNA: ribonucleic acid; DNA: deoxyribonucleic acid; siRNA: small interfering RNA; RR: ribonucleotide reductase; RRM2: ribonucleotide reductase M2; mRNA: messenger RNA; SKOV3/DDP: cisplatinresistant SKOV3 cell; RNAi: RNA interference; VEGF: vascular endothelial growth factor; MMP: matrix metalloproteinase; DMEM-F12: dulbecco modified eagle medium mix with Ham's F12 nutrient medium; FBS: fetal bovine serum; Tris-EDTA buffer: buffer containing trimethylolamine, ethylene diamine tetraacetic acid and Tween 20, PH9.0; PBS: phosphate-buffered saline. 


\section{Acknowledgments}

This research was supported by General Project of Youth Fund 2013, Affiliated Hospital of Qingdao University (1774). We thank all partners and staff who helped us in the process of this study.

\section{Competing Interests}

The authors have declared that no competing interest exists.

\section{References}

1. Siegel RL, Miller KD, Jemal A. Cancer statistics, 2018. CA Cancer J Clin. 2018;68:7-30.

2. Ribeiro J R , Schorl C, Yano N , et al. HE4 promotes collateral resistance to cisplatin and paclitaxel in ovarian cancer cells. Journal of Ovarian Research. 2016; 9:28.

3. Siddique T, Awan FR. Effects of Reg3 delta bioactive peptide on blood glucose levels and pancreatic gene expression in an alloxan-induced mouse model of diabetes. Can J Diabetes. 2016;40:198-203.

4. Ma J, Yang S, Ma A, et al. Expression of miRNA-155 in carotid atherosclerotic plaques of apolipoprotein $\mathrm{E}$ knockout (ApoE(-/-)) mice and the interventional effect of rapamycin. Int Immunopharmacol. 2017;46:70-74.

5. Zhu JD, Wang JJ, Ge G, et al. Effects of Noggin-transfected neural stem cells on neural functional recovery and underlying mechanism in rats with cerebral ischemia reperfusion injury. J Stroke Cerebrovasc Dis. 2017;26:1547-1559.

6. Duroux-Richard I, Giovannangeli C, Apparailly F. CRISPR-Cas9: A revolution in genome editing in rheumatic diseases. Joint Bone Spine. 2017;84:1-4.

7. Rahman MA, Amin AR, Wang D, et al. RRM2 regulates Bcl-2 in head and neck and lung cancers: a potential target for cancer therapy. Clin Cancer Res. 2013;19:3416-28.

8. Xia G, Wang H, Song $\mathrm{Z}$, et al. Gambogic acid sensitizes gemcitabine efficacy in pancreatic cancer by reducing the expression of ribonucleotide reductase subunit-M2 (RRM2). J Exp Clin Cancer Res. 2017;36:107.

9. Tuppurainen L, Sallinen H, Karvonen A, et al. Combined gene therapy using AdsVEGFR2 and AdsTie2 with chemotherapy reduces the growth of human ovarian cancer and formation of ascites in mice. Int J Gynecol Cancer. 2017; 27:879-86.

10. Wu X, An X, Zhang C , et al. Clb6-Cdc28 promotes ribonucleotide reductase subcellular redistribution during $\mathrm{S}$ phase. Molecular and Cellular Biology. 2018; 38: MCB.00497-17.

11. Zuckerman JE, Hsueh T, Koya RC, et al. siRNA knockdown of ribonucleotide reductase inhibits melanoma cell line proliferation alone or synergistically with temozolomide. J Invest Dermatol. 2011;131:453-60.

12. Ping C, Jian-Nong $W$, Yang $S$, et al. Gemcitabine resistance mediated by ribonucleotide reductase M2 in lung squamous cell carcinoma is reversed by GW8510 through autophagy induction. Clinical Science. 2018;132: 1417-1433.

13. Zhang M, Wang J, Yao R, et al. Small interfering RNA (siRNA)-mediated silencing of the M2 subunit of ribonucleotide reductase: a novel therapeutic strategy in ovarian cancer. Int J Gynecol Cancer. 2013;23:659-66.

14. Naito S, von Eschenbach AC, Giavazzi R, et al. Growth and metastasis of tumor cells isolated from a human renal cell carcinoma implanted into different organs of nude mice. Cancer Res. 1986;46:4109-15.

15. Wang J, Lu Z, Wang J, et al. Paclitaxel tumor priming promotes delivery and transfection of intravenous lipid-siRNA in pancreatic tumors. J Control Release. 2015;216:103-10.

16. Wong HL, Shen $\mathrm{Z}, \mathrm{Lu} \mathrm{Z}$, et al. Paclitaxel tumor-priming enhances siRNA delivery and transfection in 3-dimensional tumor cultures. Mol Pharm. 2011; 8:833-40.

17. $\mathrm{Hu} \mathrm{Q}, \mathrm{Li} \mathrm{W}, \mathrm{Hu} \mathrm{X}$, et al. Synergistic treatment of ovarian cancer by co-delivery of survivin shRNA and paclitaxel via supramolecular micellar assembly. Biomaterials. 2012;33:6580-91.

18. Sun TM, Du JZ, Yao YD, et al. Simultaneous delivery of siRNA and paclitaxel via a "two-in-one" micelleplex promotes synergistic tumor suppression. ACS Nano. 2011;5:1483-94.

19. Li J, Pang J, Liu Y, et al. Suppression of RRM2 inhibits cell proliferation, causes cell cycle arrest and promotes the apoptosis of human neuroblastoma cells and in human neuroblastoma RRM2 is suppressed following chemotherapy. Oncol Rep. 2018; 40:355-360.

20. Rasmussen RD, Gajjar MK, Tuckova L, et al. BRCA1-regulated RRM2 expression protects glioblastoma cells from endogenous replication stress and promotes tumorigenicity. Nat Commun. 2016;7:13398.

21. Roberts CM, Shahin SA, Wen W, et al. Nanoparticle delivery of siRNA against TWIST to reduce drug resistance and tumor growth in ovarian cancer models. Nanomedicine. 2017;13:965-76.

22. Kang H, Jeong JY, Song JY, et al. Notch3-specific inhibition using siRNA knockdown or GSI sensitizes paclitaxel-resistant ovarian cancer cells. Mol Carcinog. 2016;55:1196-209.
23. Shah KN, Mehta KR, Peterson D, et al. AKT-induced tamoxifen resistance is overturned by RRM2 inhibition. Mol Cancer Res. 2014;12:394-407.

24. Grunda JM, Fiveash J, Palmer CA, et al. Rationally designed pharmacogenomic treatment using concurrent capecitabine and radiotherapy for glioblastoma; gene expression profiles associated with outcome. Clin Cancer Res. 2010;16:2890-8.

25. Grolmusz VK, Karászi K, Micsik T, et al. Cell cycle dependent RRM2 may serve as proliferation marker and pharmaceutical target in adrenocortical cancer. Am J Cancer Res. 2016; 6:2041-2053.

26. Lijun $X$, Zhou B, Liu $X$, et al. Ribonucleotide reductase small subunit p53R2 facilitates p21 induction of G1 arrest under UV irradiation. Cancer Res. 2007;67:16.

27. Wang L, Meng L, Wang XW, et al. Expression of RRM1 and RRM2 as a novel prognostic marker in advanced non-small cell lung cancer receiving chemotherapy. Tumor Biol. 2014;35:1899-906.

28. Zhang $\mathrm{K}, \mathrm{Hu} \mathrm{S}, \mathrm{Wu} \mathrm{J}$, et al. Overexpression of RRM2 decreases thrombspondin-1 and increases VEGF production in human cancer cells in vitro and in vivo : implication of RRM2 in angiogenesis. Mol Cancer. 2009;8:1-12.

29. Gonzalez-Palomares B, Coronado Martin PJ, Maestro de Las Casas ML, et al. Vascular endothelial growth factor (VEGF)polymorphisms and serum VEGF levels in women with epithelial ovarian cancer, benign tumors, and healthy ovaries. Int J Gynecol Cancer. 2017;27:1088-95.

30. Dai Y, Tong R, Guo H, et al. Association of CXCR4, CCR7, VEGF-C and VEGF-D expression with lymph node metastasis in patients with cervical cancer. Eur J Obstet Gynecol Reprod Biol. 2017;214:178-83.

31. Yeh CB, Hsieh MJ, Hsieh $\mathrm{YH}$, et al. Antimetastatic effects of norcantharidin on hepatocellular carcinoma by transcriptional inhibition of MMP-9 through modulation of NF-kB activity. PLoS One. 2012;7:e31055.

32. Zhang W, Liu $Y$, Wang CW. S100A4 promotes squamous cell laryngeal cancer Hep-2 cell invasion via NF-kB/MMP-9 signal. Eur Rev Med Pharmacol Sci. 2014;18:1361.

33. Chen L, Zhang X, Chen J, et al. NF-kappa B plays a key role in microcystin-RR-induced HeLa cell proliferation and apoptosis. Toxicon. 2014; 87:120-30

34. Cunjian YI, Zhang L, Li L, et al. Establishment of an orthotopic transplantation tumor model in nude mice using a drug-resistant human ovarian cancer cell line with a high expression of c-Kit. Oncol Lett. 2014;8:2611-5. 